\title{
Depression under stress: ethical issues in genetic testing
}

Ainsley J. Newson

\section{Summary}

Genetic testing for risk of depression requires a reconsideration of ethical issues in genetics and how they manifest in psychiatric practice. A precautionary approach is advocated in that there should be limits on the use of the
5-HTT genetic test until its clinical utility and broader social impact are better understood.

\section{Declaration of interest}

None.
Ainsley J. Newson is a senior lecturer in biomedical ethics at the University of Bristol. She has degree qualifications in science, law and medical ethics, and her research examines the ethical implications of human genetics and reproduction, including family communication and prenatal diagnosis. Her PhD focused on the ethics of behavioural genetics. She teaches medical ethics to undergraduate medical students and is also Programme Manager of the MSC in Health Care Ethics and Law.

The long-heralded promises of genetic research into complex human behaviours are, it seems, coming a step closer. Increasing numbers of candidate genes associated with mental illness are being published and results replicated by other research teams. Surveys in patient and lay groups have demonstrated significant interest in accessing genetic testing to predict or partially explain one's mental health. A recent paper in the Journal provides one of the first retrospective investigations of the impact of receiving genetic information associated with a risk of depression under stress (hereinafter 'depression'). ${ }^{1}$

Yet so far, something is missing. Although the ethical implications of psychiatric genetic research and its clinical application have been discussed in the literature, this takes a generalist, prospective and speculative view. ${ }^{2-5}$ With a couple of exceptions, there is scant literature analysing the ethical implications of genetic testing for risk of depression. ${ }^{6,7}$ No detailed ethical analysis of returning research results or the clinical application of the 5-HTT gene test has been published.

This absence from the literature is interesting, given that the 5-HTT test has been possible for some time. It may be that no new ethical issues arise beyond those already debated. Considerations of genetic exceptionalism, genetic determinism, family communication and the 'right not to know' are certainly interesting but are not unique to psychiatric genetics. However, the move from Mendelian to complex genetics may necessitate a re-analysis of existing issues, integrating the ethical aspects of genetic, clinical and environmental factors. ${ }^{8}$ Psychiatry is also arguably a unique branch of medicine in that it involves the interplay between values, science and clinical judgement. ${ }^{7}$ Introducing molecular data to explain what some may see as their 'true self' could give rise to challenges to self-understanding.

\section{To whom and how should this test be offered?}

Nearly two-thirds of Wilhelm et al's cohort consented to the 5-HTT genetic test ${ }^{1}$ a higher rate than that observed for single-gene conditions such as Huntington's disease. This result is consistent with studies that predicted high levels of interest in psychiatric genetic testing. It may indicate that receiving genetic information about depression is not as threatening as for lifelimiting diseases, or that this low-risk susceptibility gene will not be overinterpreted as determinative of depression risk. However, the cohort tested were an expert group (having been involved in a longitudinal study of depression) past the mean age at onset for depression. This uptake rate may therefore represent an upper threshold.

Depending on the mechanism of access, whether through a clinic or directly to consumers, a genetic test for risk of depression could be offered as a pre-symptomatic test or as a tool to aid diagnosis. It might be offered to individuals who have experienced depression, those with a family history, or anyone at all. The test may have most relevance for those who have experienced depression, yet if individual autonomy is emphasised there is potential for any individual to choose to obtain this information to enhance their self-knowledge or to inform long-term plans. ${ }^{7}$ This wide offer may, however, cause an overemphasis on genetic factors in risk of depression.

Protocols for offering this test require consideration. First, should pre- and post-test counselling be offered or mandatory? This test provides a probabilistic result and requires careful explanation. Without significant prior knowledge, could this be achieved by telephone or online? Second, test recipients in this study were presumably able to access the test free of charge and did not have to attend clinic to obtain results. ${ }^{1}$ The test will almost certainly attract a charge in many health systems and will require rigorous evaluation to be offered through any nationally funded health service.

The test may not only be relevant to competent consenting adults. If pre-symptomatic interventions become available, the 5-HTT test could become relevant to younger people, in particular children and adolescents. ${ }^{2,6}$ It could be used as a tool for presymptomatic identification of children or young adolescents with the $s / s$ or $s / l$ genotype and to provide advice about avoiding environmental stress. But this testing regime would only be effective for population health by way of screening. Such a programme is unlikely to be introduced with present levels of efficacy and utility of this test, the lack of knowledge of geneenvironment interactions and predictability of illness onset or severity. There are also recommendations against testing children for psychiatric conditions in the absence of a clear intervention ${ }^{4}$ and unresolved ethical concerns such as the impact on the parent-child relationship and the child's developing sense of self. $^{6}$

The potential application of psychiatric genetic testing in prenatal diagnosis has also been recognised. ${ }^{2}$ Depression can be a debilitating illness and some couples with experience of this condition may wish to avoid passing it on to their children. But 
it would be premature to offer prenatal diagnosis for 5-HTT, for practical and ethical reasons. Practically, the 5-HTT gene is a measure of risk, not certainty. An s/s genotype is no indication of the certainty of depression, nor its severity. ${ }^{7}$ Ethically, there needs to be further debate on the level of absolute increase in risk of depression (if any) that would be a justifiable threshold for offering prenatal diagnosis. ${ }^{3}$ Many couples may actually be trying to avoid the stigma of the illness rather than the illness itself, which, if managed well, may not have an adverse impact on an individual's life. Public and patient education to reduce stigma is also required.

Who should offer this test should also be considered. Commercial genetic testing for some mental illnesses is already available and there is bound to be interest in this test as well. Some commercial providers offer pre- and post-test counselling; others adopt a more technical approach. Direct-to-consumer genetic testing has given rise to concerns of misinformation, but this is yet to be definitively established. Regulating commercial provision of any genetic test is difficult and is often limited to monitoring marketing and advertising. Yet given the complexity and limitations of this information, a thorough pre-test workup, establishing understanding and reinforcing the importance of reflecting on personal resilience and coping style ${ }^{1}$ should be provided face to face in a clinical environment.

\section{Mitigating adverse outcomes}

If this test is to be offered more widely, steps must be taken to ensure that adverse outcomes are monitored and minimised. Perceptions of mental health can form an important component of our sense of self. Those receiving s/s results in Wilhelm's study had significantly higher levels of distress and expectation of a future episode of depression than did those receiving $\mathrm{s} / \mathrm{l}$ or $1 / 1$ results, ${ }^{1}$ which begs the question of whether the experience of genetic testing per se could trigger a depressive episode. This potential for a 'self-fulfilling prophecy' is possible but can be mitigated by careful framing of information during pre- and post-test counselling.

The risk of genetic determinism - of attributing all health and behaviour to genetic causes - appears low from this study. Indeed, participants felt that genetics was a less important determinant of risk of depression after having the test than they did beforehand - possibly due to the careful explanation of results or the increasing public incorporation of genetic information into explanations of health and illness. ${ }^{8}$ However, further mixed-methods survey-based studies should monitor whether test recipients feel that a diagnosis is more conclusive or permanent if arising from DNA analysis than clinical diagnosis.

Privacy and confidentiality of results should also be carefully controlled and access by third parties such as insurers and employers should be strictly regulated. ${ }^{4}$ Discrimination by such bodies is by no means a certainty, but the ongoing stigmatisation of mental illness ${ }^{9}$ means that policy-makers cannot become complacent. $^{3}$

\section{Future directions}

The 5-HTT gene is unlikely to become the sine qua non to managing depression. However, if genetic testing continues, I would advocate adopting a precautionary approach, to monitor outcomes and further investigate clinical utility. This approach comprises a number of claims. Although determinism and discrimination are unlikely and no recipients of this test regretted their decision, given a lack of clear medical benefit the test should initially be used to inform clinical management in those who have experienced depression, rather than as a pre-symptomatic or predictive test. ${ }^{4}$ It should be offered in a clinical environment to competent consenting individuals who have had access to validated pre-test information and receive post-test follow-up. Testing using direct-to-consumer methods should be discouraged and marketing should be carefully monitored. The test should not be offered to people experiencing an acute episode of depression, as receipt of the test result may later be associated with that depressive mood and the result may hinder recovery. Testing in younger children and for prenatal diagnosis is not currently supported by clinical evidence or ethical arguments and will have little predictive value. Population screening for depression may be beneficial but the 5-HTT test is unlikely to be the best mechanism for this. Testing should also be accompanied by ongoing mixedmethods survey research to examine post-test adjustment and engagement in ethical discussion by all stakeholders.

Ainsley J. Newson, PhD, Centre for Ethics in Medicine, University of Bristol, 3rd Floor, Hampton House, Cotham Hill, Bristol BS6 6AU, UK.

Email: ainsley.newson@bristol.ac.uk

First received 30 Jan 2009, final revision 12 Feb 2009, accepted 13 Feb 2009

\section{References}

1 Wilhelm K, Meiser B, Mitchell PB, Finch AW, Siegel J, Parker G, et al. Issues concerning feedback to participants about their serotonin transporter genotype and risks for depression. Br J Psychiatry 2009; 194: 404-10.

2 Appelbaum PS. Ethical issues in psychiatric genetics. J Psychiatr Pract 2004; 10: $343-51$

3 Hoop JG. Ethical considerations in psychiatric genetics. Harv Rev Psychiatry 2008; 16: 322-38.

4 Nuffield Council on Bioethics. Mental Disorders and Genetics: The Ethical Context. Nuffield Council on Bioethics, 1998 (http:// www.nuffieldbioethics.org/go/ourwork/mentaldisorders/ publication_300.html).

5 Dinwiddie $\mathrm{SH}$, Hoop J, Gershon ES. Ethical issues in the use of genetic information. Int Rev Psychiatry 2004; 16: 320-8.

6 Morley KI, Hall WD, Carter L. Genetic screening for susceptibility to depression: can we and should we? Aust N Z J Psychiatry 2004; 38: 73-80.

7 Wallace J. Ethics and psychiatric genetics: is it ethical to test for 'depression genes'? Psychiatr Bull 2004; 28: 279-80.

8 Foster MW, Royal CDM, Sharp RR. The routinisation of genomics and genetics: implications for ethical practices. J Med Ethics 2006; 32: 635-8.

9 Van 't Veer JTB, Kraan HF, Drosseart HC, Modde JM. Determinants that shape public attitudes towards the mentally ill: a Dutch public study. Soc Psychiatry Psychiatr Epidemiol 2006; 41: 310-7. 